Department of Health and Human Services.

\section{HIV-Infected Scientist Claims AIDS Vaccine Does Not Work}

A French scientist going by the pseudonym of Philip Bertrand said that a prototype of a vaccine that is about to undergo human testing in the U.S. failed to halt the progression of his human immunodeficiency virus (HIV) infection. While conducting research in Africa, Bertrand claims he received the vaccine one week after he was infected with HIV when he accidentally cut himself on a glass tube that contained HIV. Bertrand said the vaccine was being developed under the direction of National Institutes of Health (NIH) researcher Dr. Robert Gallo and was sent to French scientist Dr. Daniel Zagury in Zaire for human testing. Bertrand charges that Gallo and Zagury knowingly omitted relevant facts about the vaccine's performance from a journal article they published concerning the efficacy of the vaccine in humans. Specifically, the article did not reveal that Bertrand had been infected with HIV before receiving the vaccine or that antibodies found in Bertrand's cells could be a result of contact with HIV rather than the vaccine.

A similar vaccine has been approved for human testing in the United States. Drs. Gallo and Zagury and an NIH spokesperson declined to comment.

FROM: Chicago Tribune. September 5, 1993.

\section{CDC Offers Guidance on Drug- Resistant TB}

The spread of drug-resistant tuberculosis (TB) has prompted the CDC to issue new treatment recommendations to curb further transmission. In a special report, the CDC advises the use of a four-drug regimen of isoniazid, rifampin, pyrazinamide, and either streptomycin or ethambutol for the initial empiric treatment of TB. The agency also says that directly observed therapy (DOT) should be considered for all TB patients because failure to take a full course of medication is the major factor in development of drug resistance. Critics of the new CDC guidelines say that DOT should be mandated, not "considered."

The new recommendations also call for in vitro drug susceptibility testing on the first isolate of $\mathrm{Myco-}$ bacterium tuberculosis in all patients with TB to provide the basis for clinical therapeutic decisions, reporting the results to public health authorities. In addition, drug susceptibility testing will be important for identifying emerging drug resistance and helping to monitor control efforts in areas where resistance already is established.

'The new four-drug regimen and susceptibility testing go hand-in-hand," said Larry Geiter, chief of clinical research in the CDC's Tuberculosis Elimination Division. 'These new guidelines add a fourth drug to the previously recommended three-drug regimen. Pyrazinamide previously had been added to the TB drugs of choice, isoniazid and rifampin. Pyrazinamide allowed us to cut back to a six-month regimen, as opposed to nine months, and cuts the relapse rate to below 5\%."

A four-drug regimen can be administered intermittently instead of daily. It is effective when given three times a week from the beginning of therapy or twice a week following an initial two-week phase of daily therapy. Clinicians should consider the three-drug regimen acceptable only in areas where isoniazid resistance is less than $4 \%$.

Announcement of this recommendation also coincides with the renewed manufacture of streptomycin in the United States. After a two-year absence due to sterility problems in bulk supplies from a foreign manufacturer, Pfizer Pharmaceuticals Inc was to resume domestic production on July 6, 1993.

Drug resistance has added a chilling dimension to the recent resurgence of TB. In New York City, for example, $33 \%$ of TB cases were resistant to at least one drug, and 19\% were resistant to both isoniazid (INH) and rifampin (RIF). Among recurrent cases of TB nationwide, $6.9 \%$ were resistant to both INH and RIF in 1991, compared with 3\% during the period 1982 to 1986 , according to the CDC.

These recommendations update previous CDC and American Thoracic Society recommendations for treatment of TB.

FROM: Centers for Disease Control and Prevention. Initial therapy for tuberculosis in the era of multidrug resistance: recommendations of the Advisory Council for the Elimination of Tuberculosis. M M WR 1993;42 (RR-7) :1-8.

\section{Joint Commission to Make Hospital Performance Information Available to Public}

Starting next year, the Joint Commission on Accreditation of Healthcare Organizations for the first time will release information to the public detailing how hospitals meet specific performance standards. Joint Commission President Dr. Dennis O'Leary says, 'This is a landmark issue for us, and it is very much in line with the reform environment. The change in the Joint Commission's confidentiality and disclosure policy, approved recently by the commission's board, recognizes the accrediting agency's obligation to share information with patients, purchasers, and other stakeholders in healthcare delivery systems."

Under the new policy, standards-compliance rat- 